\title{
SISTEMA DE INFERENCIA DIFUSA PARA LA MODIFICACIÓN DE COLOR AJUSTADO EMPLEANDO ENJAMBRE DE PARTÍCULAS
}

\author{
FUZZY INFERENCE SYSTEM FOR THE AMENDMENT \\ OF ADJUSTED COLOR USING PARTICLE SWARM
}

Linda Sheriyn Rodríguez Castro ${ }^{1}$, Iván Javier Ponce Fajardo ${ }^{2}$, Helbert Eduardo Espitia Cuchango 3

${ }^{1}$ Universidad Distrital Francisco José de Caldas, Bogotá, Colombia. E-mail: lsrodriguezc@correo.udistrital.edu.co
${ }^{2}$ Universidad Distrital Francisco José de Caldas. Bogotá, Colombia. E-mail: ijponcef@correo.udistrital.edu.co
${ }^{3}$ Helbert Eduardo Espitia Cuchango,Universidad Distrital Francisco José de Caldas. Bogotá, Colombia.
E-mail:heespitiac@udistrital.edu.co
*Correspondencia del autor. Linda Sheriyn Rodríguez Castro. Universidad Distrital Francisco José de Caldas. Bogotá, Colombia.
E-mail: 1srodriguezc@correo.udistrital.edu.co

\section{RESUMEN}

En el presente artículo se propone un sistema de inferencia difusa para la manipulación del color en imágenes digitales para cambiar su estilo artístico al arte psicodélico. Inicialmente mediante conjuntos y reglas de inferencia difusas se produce imágenes con colores característicos de este tipo de arte. Posteriormente se ajusta el sistema de inferencia propuesto con la técnica denominada optimización basada en enjambres de partículas evidenciando mejora en los resultados obtenidos.

Palabras claves: Arte evolutivo, arte psicodélico, colorimetría, lógica difusa, PSO, procesamiento de imágenes.

\begin{abstract}
This paper proposes a fuzzy inference system for color manipulation in digital images to change the art style to psychedelic art. Initially through sets and fuzzy inference rules, generating images with colors characteristic of this type of art. Later fits inference system with the technique proposed an optimization algorithm based on particle swarms evidencing improved results.
\end{abstract}

Key Words: Evolutionary art, psychedelic art, colorimetry, fuzzy logic, PSO, image processing. 
Sistema de inferencia difusa para la modificación de color ajustado empleando enjambre de partículas. Rodríguez et al.

\section{INTRODUCCIÓN}

El arte evolutivo es una línea de investigación que asocia al campo del arte y la computación. Consiste en traducir los procesos humanos de creatividad en algoritmos computacionales (1) los cuales pueden ser verificados por expertos u otros sistemas buscando además automatizar los sistemas de evaluación (2).

Se han adelantado bastantes desarrollos particularmente en el tratamiento del color, detectando estructuras especificas en las imágenes, algunos de estos son: cambio automático en el color de los subtítulos en películas a color (3); la detección del fuego imágenes captadas por cámaras de seguridad (4); reconocimiento de objetos por color en fotografías para mejorar las búsquedas web (5); reducción de ruido en imágenes a color (6); optimización de procesamiento multimedia para reducir la complejidad al representar color verdadero (7); clasificación de color por segmentación de imágenes y algoritmos inspirados en enjambres para reducir el error (8).

El arte y el manejo del color no pueden tratarse mediante técnicas que establezcan límites cerrados y dicotómicamente excluyentes como la lógica clásica. Esto llevaría a clasificaciones taxonómicas estrictas que harían pensar en colores que pertenecen o no a una categoría mientras se excluyen de otra (9). La lógica difusa, por su parte, permite manejar la incertidumbre existente en sistemas donde una clasificación no es inmediata ni cerrada (10). Al establecer reglas $\mathrm{y}$ conjuntos con rangos de pertenencia que difuminan las fronteras, permitiendo en el caso del tratamiento del color términos como "verde-rojizo" (11).

Para apoyar el uso de la lógica difusa en la percepción del color, se viable adoptar el modelo de color RGB. Pues permite clasificar el color a través de códigos que en tres diferentes conjuntos: rojo, verde y azul (16). Algunos trabajos, aunque relacionados en menor medida, es el reconocimiento de lenguaje natural para identificar el contexto de un paisaje y colorear de forma automática un cuadro (12); igualmente avances en el mapeo para la composición audiovisual y de síntesis de sonido que se introducen en el campo del arte evolutivo.

La propuesta desarrollada en este artículo tiene como eje central el procesamiento de imágenes, para la identificación de color y modificación de su expresión artística. En particular, el tratamiento visual se genera con la abstracción de características del arte denominado psicodélico. El término psicodélico fue acuñado por el psicólogo británico Humphry Osmond, quien afirmaba que su significado refería "lo que manifiesta la mente" (13). En especial, la pintura presenta visiones del mundo no convencionales y propiedades como uso de patrones fractales, colores brillantes y con alto contraste, profundidad en los detalles, formas de tipo radial, espirales. Por tal motivo, la pintura psicodélica es adecuada para evidenciar la transformación en los resultados de la implementación del presente artículo.

Atendiendo el carácter propio del estilo artístico descrito y el modelo de inferencia difusa para la transformación de color, se propone: variables de entrada y salida, reglas lógicas condicionales y funciones de pertenencia, que conlleven a la modificación de los colores en las imágenes digitales para adquirir los efectos deseados. Este trabajo constituye un primer paso hacia sistemas de modificación visual no acotados a un único estilo artístico.

\section{Sistema de lógica difusa}

La lógica difusa es una técnica de inteligencia computacional caracterizada por manejar la incertidumbre y la ambigüedad del mundo real. La vaguedad se relaciona con el conocimiento del valor de una función en términos de una variable con valor conocido (14), (17).

La fusificación es el primer paso que se realiza un modelo de lógica difusa. Este cosiste en obtener las variables de entrada y salida junto con las funciones de pertenencia que describen su comportamiento. El segundo paso de denomina inferencia lógica donde es determinan las reglas que levan los datos de entrada a las salidas determinadas. El tercer y última paso es el proceso de defusificación, adecúa los datos obtenidos (valores difusos) a valores reales mediante la utilización de métodos como el Centroide o Promedio Ponderado (19).

\section{Sistema de inferencia difusa propuesto para la modificación del color}

A continuación se detalla el proceso realizado para obtener el sistema de lógica difusa que posteriormente se optimiza. 


\subsection{Descripción del problema}

Existe ambigüedad en la selección de colores de un estilo artístico particular, por ejemplo el psicodélico. Aun más si a partir de estos se desea cambiar la sensación visual de una imagen digital. Por tanto se hace necesaria la definición de rangos de color. Dichos rangos pueden ser identificados a través de obras digitales mundialmente conocidas y producidas por artistas dentro del arte psicodélico.

Un claro ejemplo de obras de arte que marcan el objetivo a seguir se observa en la Figura 1 y la Figura 2.

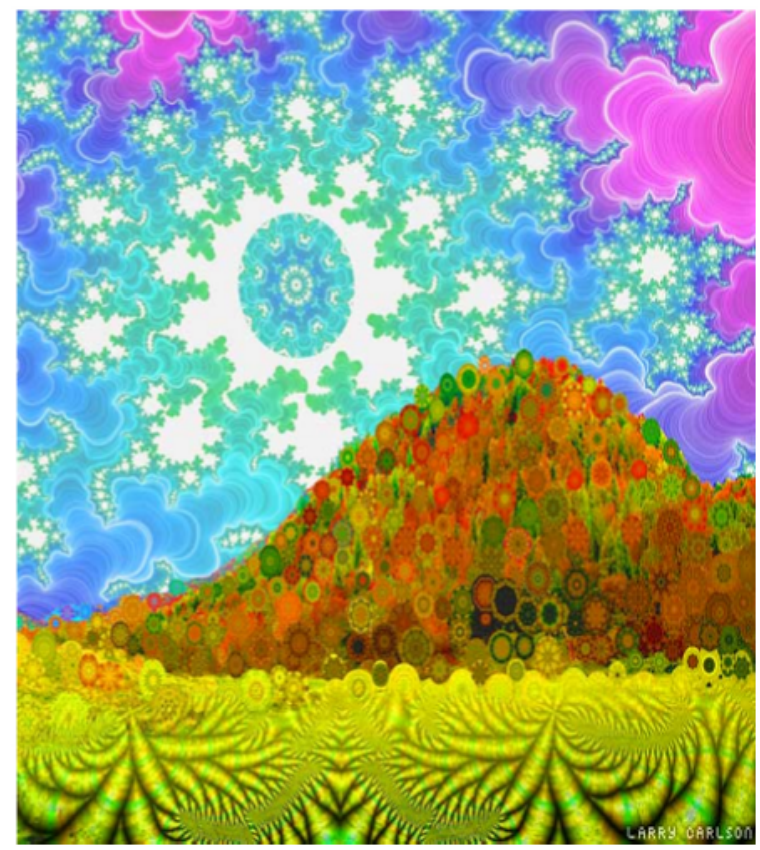

Figura 1. Electrix Sunshine. Autor: Larry Carlson (2006).

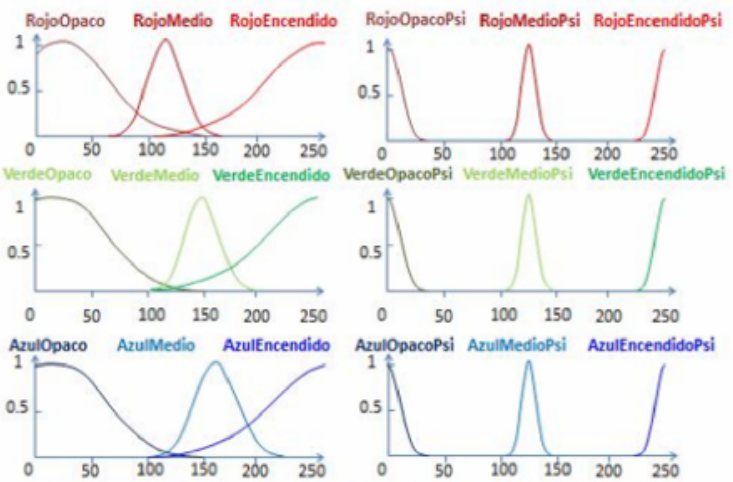

(a) Funciones de Pertenencia del Sistema Difuso sin Optimizar

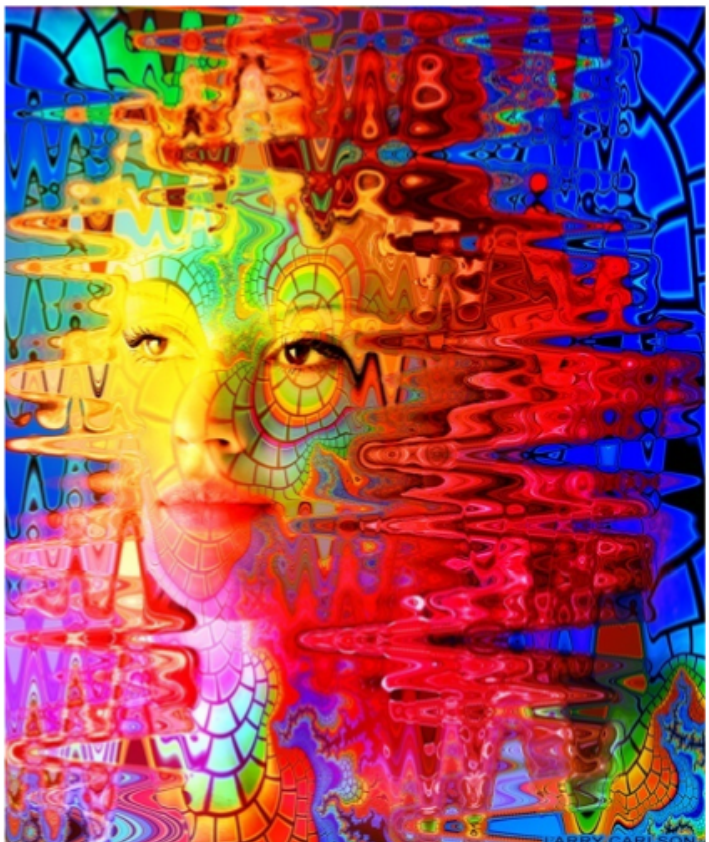

Figura 2. Electrix Eyes. Autor: Larry Carlson (2014).

reglas de inferencias.

\subsection{Funciones de pertenencia}

Dado que se pretende transformar la sensación visual una imagen digital en una percepción psicodélica, se definió leer pixel por pixel la imagen a modificar. Para esto se establecieron tres variables, una por cada componente del modelo RGB, es decir rojo, verde y azul, que pueden variar en un rango de 0 a 255 .

Cada variable tanto de entrada como de salida posee tres funciones de pertenecía de tipo gaussiana para

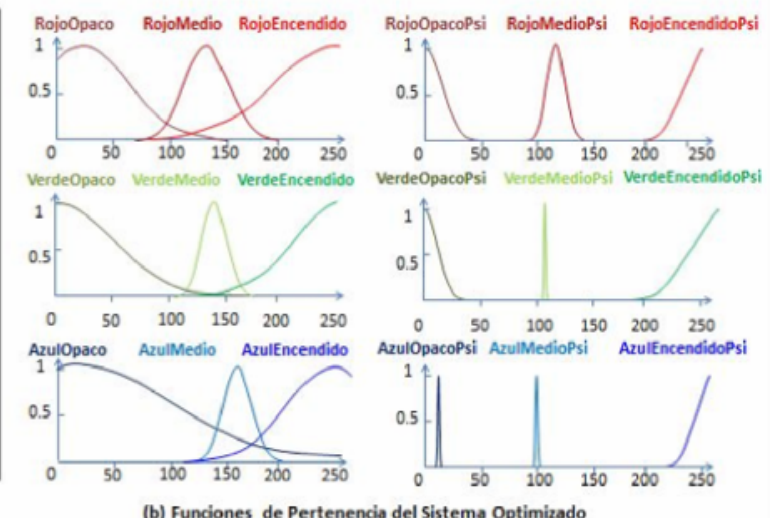

(b) Funciones de Perten
Como puede detallarse los colores predominantes son diferentes tonalidades de azules, morados, fucsias, verdes y naranjas. Basándose en estas observaciones junto con otros ejemplos observados se obtuvieron las denotar tonos oscuros, medios y claros como se observa en la Figura 3 (a). La disposición de las variables salida es menor con el fin de obtener cierta gama de colores más limitada y acotada por las características definidas. 
Sistema de inferencia difusa para la modificación de color ajustado empleando enjambre de partículas. Rodríguez et al.

\subsection{Reglas}

Una vez establecidos los conjuntos difusos, se determina cuales tonalidades serán reemplazadas y que combinación de estos llevarán a tal resultado. En la Tabla 1 se consignan la totalidad de las reglas establecidas.
La optimización basada en enjambres de partículas PSO (Particle Swarm Optimization) fue propuesta originalmente por James Kennedy y Russell Eberhart (18). Este algoritmo se encuentra basado en el comportamiento social de bandadas de aves. Los pasos básicos de un algoritmo PSO son los siguientes:

- Inicializar el enjambre en el espacio solución.

Tabla 1. Reglas de inferencia para el sistema difuso. Fuente: Autores.

\begin{tabular}{|c|c|c|c|c|c|c|c|c|}
\hline \multirow[b]{2}{*}{$\mathbf{N}$} & \multicolumn{5}{|c|}{ Antecedente } & \multicolumn{3}{|c|}{ Consecuente } \\
\hline & Rojo & Op. & Verde & Op. & Azul & Rojo & Verde & Azul \\
\hline 1 & Encendido & And & Opaco & And & Opaco & Encendido & Opaco & Opaco \\
\hline 2 & Opaco & And & Encendido & And & Opaco & Opaco & Encendido & Opaco \\
\hline 3 & Opaco & And & Opaco & And & Encendido & Opaco & Opaco & Encendido \\
\hline 4 & Opaco & And & Opaco & And & Opaco & Opaco & Opaco & Opaco \\
\hline 5 & Encendio & And & Encendido & And & Encendido & Encendido & Encendido & Encendido \\
\hline 6 & Medio & And & Medio & And & Encendido & Opaco & Opaco & Encendido \\
\hline 7 & Opaco & And & Encendido & And & Encendido & None & Medio & Encendido \\
\hline 8 & Medio & And & Opaco & And & Opaco & Encendido & Medio & Opaco \\
\hline 9 & Medio & And & Opaco & And & Opaco & Encendido & Opaco & Opaco \\
\hline 10 & Encendido & And & Medio & And & Medio & Encendido & Opaco & Medio \\
\hline 11 & Medio & And & Medio & And & Opaco & Encendido & Encendido & Opaco \\
\hline 12 & Medio & And & Opaco & And & Encendido & Encendido & Opaco & Encendido \\
\hline 13 & Medio & And & Encendido & And & Opaco & Opaco & Encendido & Opaco \\
\hline 14 & Encendido & And & Encendido & And & Opaco & Encendido & Encendido & Opaco \\
\hline 15 & Encnedido & And & Opaco & And & Encendido & Encendido & Opaco & Encendido \\
\hline 16 & Medio & And & Opaco & And & Medio & Encendido & Opaco & Encendido \\
\hline 17 & Opaco & And & Medio & And & Medio & Opaco & Encendido & Encendido \\
\hline
\end{tabular}

A manera de ejemplo, en la regla número 10 el antecedente representa tonalidad de color rosado, dado por el producto de una componente roja de tipo rojo encendido, una componente verde de tipo verde medio y una componente azul de tipo azul medio. Lo cual el consecuente lo transformará en tonalidades fucsia.

\section{Ajuste del sistema}

La optimización basada en enjambre de partículas se encuentra basada en el comportamiento de enjambres de animales como aves y peces, por tanto se dice que es una optimización bio-inspirada.
- Evaluar el desempeño de cada individuo.

- Encontrar el mejor desempeño individual y colectivo.

- Determinar la velocidad de cada partícula.

- Realizar el desplazamiento de cada individuo a la nueva posición.

- Repetir desde el paso 2 hasta cumplir algún criterio de finalización.

Un algoritmo básico PSO (Particle Swarm Optimization) se puede apreciar en la Figura 4. 
Revista de Investigaciones - Universidad del Quindío

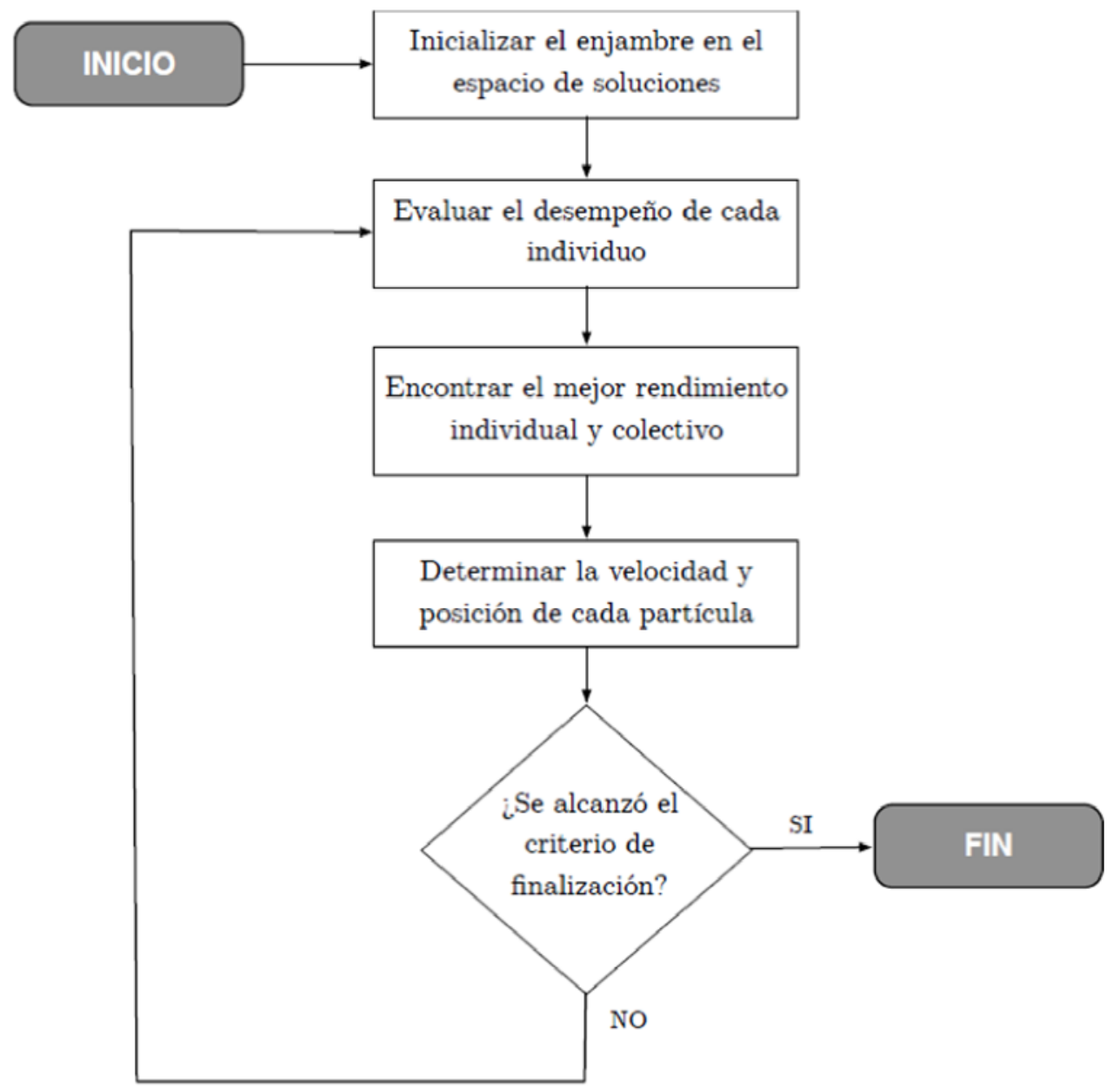

Figura 4. Diagrama de Flujo Algoritmo PSO. Fuente: Autores.

Un algoritmo básico PSO está regido por las ecuaciones de velocidad y posición. Para la velocidad de cada individuo se tiene:

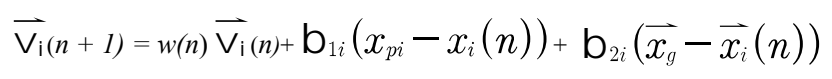

La posición de calcula mediante (2):

$$
\overrightarrow{x_{i}}(n+1)=\overrightarrow{x_{i}}(n)+\overrightarrow{\mathbf{O}_{i}}(n+1)
$$

Siendo:

(1) $\beta_{1} y \beta_{2}$

- $\quad i$

Es el índice de cada individuo

- $n$

Es el índice de tiempo discreto (itera ción)

- $\overrightarrow{\mathrm{V}_{\mathrm{i}}}$

- $\vec{x}_{i}$

- $w(n)$

Es la velocidad del i-ésimo individuo

- $p i$

Es la posición del i-ésimo individuo

Es la función de inercia

Es la mejor evaluación encontrada para el i-ésimo individuo

- $g$ Es la mejor evaluación encontrada para el enjambre

- $\overrightarrow{x_{p i}} \quad$ Es la mejor posición encontrada para el i-ésimo individuo
Es la mejor posición encontrada para el enjambre

Son números aleatorios en el rango de $[0,1]$

Aunque se pueden tener diferentes enfoque para el factor de inercia (20), en esta aplicación el factor de inercia se considera de decrecimiento lineal de la forma:

$$
w(n)=w_{\max }-\frac{W_{\max }-W_{\min }}{n} n
$$

Donde $w_{\max } y w_{\text {min }}$ son los valores máximos y mínimos de inercia y $N$ el número total de iteraciones.

Mediante la utilización de la optimización bio-inspirada en este caso la optimización basada en enjambre se mejora el sistema difuso ajustando los valores de media de la distribución y la desviación típica asociados a las funciones de pertenencia de cada variable.

La optimización del sistema utiliza el error expresado en la información de una función objetivo o índice de 
desempeño. Esta función de desempeño se obtiene de comparar los datos resultantes del sistema propuesto o datos simulados contra datos reales suministrados por expertos en el campo del arte. Los datos reales se obtuvieron con la distinción de colores expresados en código RGB, ubicados a lo largo de todo el espectro de color, a partir de los cuales los expertos generaron su equivalente en el estilo artístico psicodélico. Tanto los datos reales como los simulados comparten la misma información de entrada correspondiente a los casos de prueba mencionados.

Una vez se cuenta con la función a optimizar, y teniendo en cuenta que esta función representa el error generado de la estimación de valores para los rangos de color, es importante aclarar que la optimización a aplicar para el ajuste del sistema es una minimización. Para definir los principales parámetros que usa el algoritmo de optimización basado en enjambres de partículas se considera la definición de un vector de 36 posiciones (número total de variables asociadas a las 18 funciones de pertenencia que incluyen las entradas y salidas) tomadas del sistema propuesto. Dado este vector se conforma el rango de búsqueda en el que las partículas se situarán. Este rango corresponde a una matriz de $2 \times 36$ posiciones donde la primera columna corresponde al valor menos 25 unidades de cada posición del vector y la segunda columna corresponde a la suma de 25 unidades a cada valor del vector. Las 25 unidades se determinaron como región de holgura viable puesto que el rango donde debe oscilar los parámetros a optimizar es entre 0 y 255 (rango del código RGB) aproximando a un 10\% de este rango. De igual forma, la optimización retorna los nuevos valores de los parámetros de las funciones de pertenencia en un nuevo vector de igual tamaño utilizado para construir el sistema difuso mejorado. Con la ayuda del software MATLAB $\AA$, se ejecuta 50 veces el algoritmo de optimización generando esta misma cantidad de posibles soluciones. Por lo cual se realizó un análisis para seleccionar la mejor de las soluciones obtenidas. En la Figura 3 (b) se observa el ajuste de las funciones de pertenencia optimizadas.

\section{Resultados}

Se implementó un algoritmo que recorre uno a uno los pixeles de una imagen de entrada como si se tratase de una matriz. Cada uno de los datos obtenidos de la imagen se ingresan en el sistema difuso sin optimizar y optimizado, obteniendo una nueva imagen de salida que pertenece al estilo psicodélico. En Figura 5 y la Figura 6 se presenta en la parte superior la imagen original ingresada al sistema difuso; en la parte inferior izquierda se observa el resultado generado luego aplicar el sistema difuso sin optimizar, es decir con las funciones de pertenencia expuestas en la Figura 3 (a) y en la parte inferir derecha se expone el resultado de la aplicación del sistema difuso optimizado, cuyas funciones de pertenencia son las presentadas en la Figura 3 (b).

El tiempo promedio de cómputo para la ejecución de los sistemas difusos teniendo de entrada una imagen de 1024 x 760 pixeles es de 50 a 70 minutos. Se espera que el tiempo para imágenes de menor tamaño no supere los 25 minutos.

De los resultados se infiere que para el sistema difuso optimizado se observan mejoras notables como la continuidad en el color, la conservación de detalles de la imagen original y la intensidad de las tonalidades, aspectos no tan evidentes en el sistema difuso inicial.
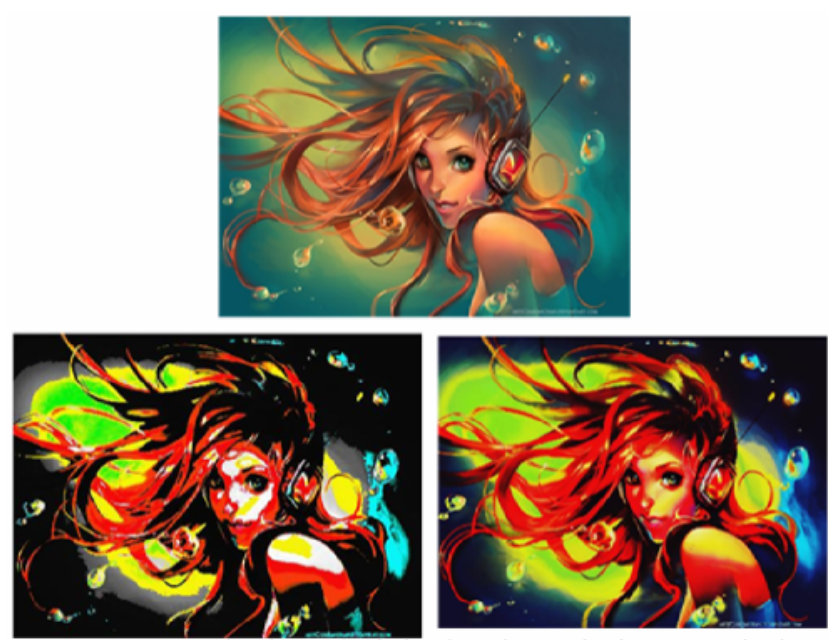

Figura 5. Resultado 1. (original - sin optimizar - optimizado). Seres de Fantasia Vol 2: Sakimichan (C.
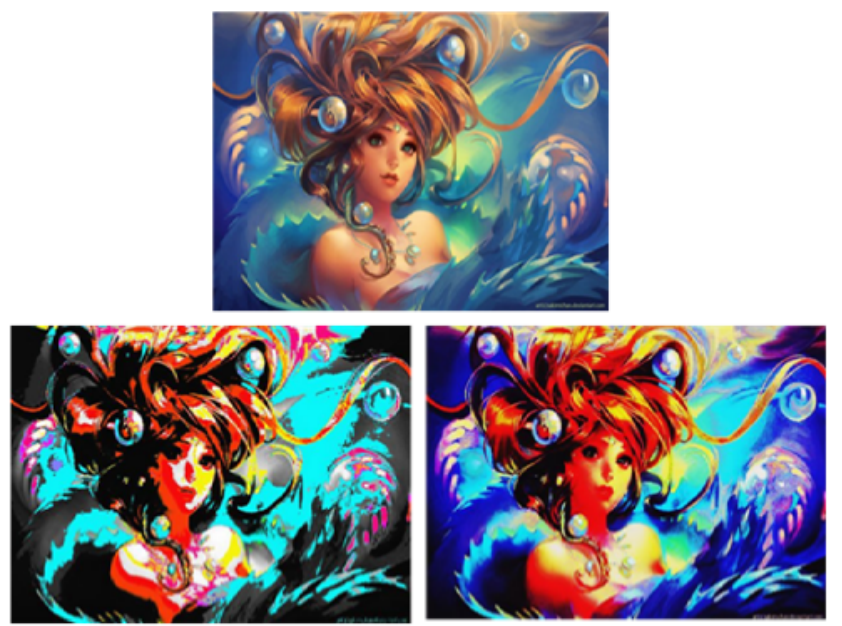

Figura 6. Resultado 2. (original - sin optimizar - optimizado). Seres de Fantasia Vol 2: Sakimichan (C). 


\section{Conclusiones}

El proceso de investigación orientado por los expertos en el campo del arte permitió establecer que las tonalidades encendidas o tonos altos constituyen la escala de colores del estilo gráfico psicodélico. La transformación a este tipo de tonalidades puede realizarse mediante la saturación de colores que poseen una o dos componentes (del código de color) con valores mayores a las demás. Se pude observar el resultado de la aplicación de esta estrategia en la Tabla 2.

Este trabajo constituye una aproximación al campo del arte evolutivo, sirviendo de base a posibles desarrollos donde su objetivo incluya la modificación de la forma en imágenes digitales (apoyándose en estilos artísticos como el optical art y el arte cinético).

Para futuros trabajos que involucren el manejo del color se espera trabajar en un algoritmo inteligente para la detección de regiones con colores similares para su transformación.

Futuras aplicaciones del presente trabajo incluyen la posibilidad de su uso en suites de diseño gráfico, para el cambio del estilo artístico con nuevos efectos visuales a un bajo costo computacional. La aplicación presentada y desarrollada puede ser de utilidad para campos como la publicidad y el diseño, entre otros.

\section{Reconocimientos}

Los autores agradecen a los profesores de la facultad de Artes de la Universidad Distrital Ricardo Forero Cárdenas y Alberto Díaz Lara por su orientación en el campo artístico y manejo del color digital. Además, agradecen especialmente al artista Larry Carlson, por permitir el uso de algunas de sus obras artísticas, su colaboración apoyó en gran medida el desarrollo del presente artículo.

\section{BIBLIOGRAFÍA}

1. Steve R. DiPaola. Incorporating characteristics of human creativity into an evolutionary art algorithm. 2007; 10(2), 97-110.

2. Yang Li. Adaptive Learning Evaluation Model For Evolutionary Art. Brisbane, Australia. WCCI 2012, IEEE World Congress on Computational Intelligence June. 2012; 1 - 8.

3. Davoudi, M. y SeifNaraghi, N. Adaptive Subtitle and Caption Coloring Using Fuzzy Analysis. World Congress on Computer Science and Information Engineering. 2009; 764 - 768.

4. Celik, T., Ö zkaramanl, H., Demirel, H.. Fire Pixel Classification using Fuzzy Logic and Statistical Color Model. ICASSP 2007. IEEE International Conference on Acoustics. 2007; Pág: I-1205 - I-1208.

5. Millet, c., Bloch, I., Popescu, A. Using the Knowledge of Object Colors to Segment Images and Improve Web Image Search. 2007; 569-584.

6. Yuksel, M. y Basturk, A. Application of Type-2 Fuzzy Logic Filtering to Reduce Noise in Color Images. Erciyes University, TURKEY. IEEE Computational Intelligence Magazine, 2012; 7(3), 25-35.

7. FuChung, F. y Fung, B. Fuzzy Color Quantization and its Application to Scene Change Detection. 2003.

8. Borji, and M. Hamidi. Evolving a Fuzzy Rule-Base for Image Segmentation. International Journal of Intelligent Technology . 2007; 2 (3) 471-476.

9. P.B. Khanale and A.B. Kurhe. Color Perception of Images Using Fuzzy Logic. Advances in Computational Sciences and Technology. 2011; 4(1) 1-8.

10. Jung-Min, O., Bang, B., Lee, G. Personal Color Decision System Using Fuzzy Logic. International Conference on Convergence and Hybrid Information Technology 2008; 790-795.

11. Universidad de Extremadura. Aplicaciones de la lógica difusa a la colorimetría. Revista "Hiperenciclopédica" de Divulgación del Saber. 2012; 6(4).

12. Terano, T., Masui, S., Watanabe, H. Coloring of a Landscape by Fuzzy Logic. Hosei University, Kajino cho, Koganei, Tokyo 184, Japan. IEEE International Conference Fuzzy Systems. 1992; 13-20.

13. Luft, Eric. Die at the Right Time! A Subjective Cultural History of the American Sixties. 1952.

14. D'Negri, C. y De Vitro, E. Introducción al Razonamiento Aproximado: lógica difusa. Revista Argentina de Medicina Respiratoria. 2006; 4, 126-136. 
Sistema de inferencia difusa para la modificación de color ajustado empleando enjambre de partículas. Rodríguez et al.

15. Enrique, A. Laguna, M. y Martí, R. Métodos Evolutivos. Revista Ingenieria UC 2003; 10(3) 80-89.

16. Young, T. Bakerian Lecture: On the Theory of Light and Colours. Phil. Trans. R. Soc. 1802; 12-48.

17. Zadeh, L. Fuzzy Sets. Information and Control. 1965.; 338-353.

18. R. Eberhart, J. Kennedy, "Particle swarm optimization”. IEEE Proceedings Neural Networks. 1995.

19. Ramírez, Osvaldo. Simulaci'on en simmechanics de un sistema de control difuso para el robot udlap. 2008.

20. Bansal J. C., Singh P. K., Saraswat Mukesh, Verma Abhishek, Jadon Shimpi Singh, Abraham Ajith. Inertia Weight Strategies in Particle Swarm Optimization. IEEE Third World Congress on Nature and Biologically Inspired Computing. 2011. 